\title{
Quadratic Solitons with Gain and Loss
}

\author{
Lluis Torner, Member, IEEE, Jochen Dörring, and Juan P. Torres
}

\begin{abstract}
Propagation of soliton signals in quadratic nonlinear media under conditions for second-harmonic generation in the presence of multiple-frequency-band absorption and amplification is addressed. The reshaping and energy redistribution of the multiple-frequency solitons under the action of the singleband loss and gain is investigated and shown in detail. New chirped localized solitary-wave solutions that exactly compensate loss and gain are presented. Practical implementation conditions are discussed.
\end{abstract}

Index Terms-Erbium, frequency conversion, nonlinear optics, self-focusing, solitons.

\section{INTRODUCTION}

O PTICAL solitons in quadratic nonlinear media constitute an active area of research. Interest is driven by the new phenomena that are continuously uncovered, and by their widespread potential applications (reviewed in [1]-[3]). Quadratic solitons are formed by the mutual trapping between the several waves that parametrically interact in the crystal, and they are stable both in waveguides and in higher dimensional configurations, including multiple-pass geometries, in settings for the spatial, temporal, and spatio-temporal trapping of light. Potential applications include a variety of beam and pulse manipulation schemes, all-optical signal processing devices, laser systems containing quadratic nonlinear crystals, and photonic quantum devices.

However, quadratic solitons are intrinsically multiple-wave multiple-frequency entities. Therefore, their experimental formation and potential technological exploitation requires suitable materials and operation conditions that exhibit low absorption losses at all the wavelength bands involved. This corresponds to two bands in the simplest case of secondharmonic generation (SHG) and up to three bands in the general case of optical parametric amplification (OPA) and optical parametric oscillation (OPO). Such conditions can be met with existing high-quality materials; thus, spatial solitons have been observed with picosecond pulses in planar waveguides made of lithium niobate $\left(\mathrm{LiNbO}_{3}\right)$ cut for type I phase-matching SHG pumped at $\lambda=1.32 \mu \mathrm{m}$ [4], in up- and down-conversion SHG schemes in bulk potassium titanyl phosphate $\left(\mathrm{KTiOPO}_{4}\right.$ or KTP) cut for type II phasematching at $\lambda=1.064 \mu \mathrm{m} \mathrm{[5],} \mathrm{[6],} \mathrm{in} \mathrm{potassium} \mathrm{niobate}$ $\left(\mathrm{KNbO}_{3}\right)$ cut for type I noncritically phase-matched SHG at

Manuscript received March 12, 1999; revised June 3, 1999. This work was supported by the Spanish Government under Contract PB95-0768 and by the European Union and the Centre de Supercomputacio de Catalunya through the TMR programme.

The authors are with the Laboratory of Photonics, Department of Signal Theory and Communications, Universitat Politecnica de Catalunya, Barcelona ES 08034, Spain.

Publisher Item Identifier S 0018-9197(99)05965-5. $\lambda=985 \mu \mathrm{m}$ [7], in quasi-phase-matched SHG in periodically poled $\mathrm{LiNbO}_{3}$ (PPLN) pumped at $\lambda=1.064 \mu \mathrm{m}$ [8], and in OPA in lithium triborate $\left(\mathrm{LiB}_{3} \mathrm{O}_{5}\right)$ operated for type I noncritical phase-matching at the signal-idler band $\lambda=0.67-$ $2.5 \mu \mathrm{m}$ [9]. Pulse narrowing consistent with temporal soliton formation was observed recently using achromatic phasematching techniques in $\beta$-barium borate $\left(\beta-\mathrm{BaB}_{2} \mathrm{O}_{4}\right.$ or $\left.\mathrm{BBO}\right)$ pumped with 200-fs pulses for SHG at $\lambda=0.527 \mu \mathrm{m}$ [10].

In this paper, we address the possibility to form quadratic solitons in new settings, unexplored wavelength bands, and novel materials where the different waves forming the solitons experience significant absorption losses or linear gain depending on the wavelength band to which they belong. We focus on the simpler case of SHG, but qualitative results and tendencies are expected to hold for all parametric processes. First, we show the impact of single-frequency absorption on the decay of the multiple-wave solitons as a function of the light energy flow and of the existing wavevector mismatch between the interacting waves. Second, we show that, under appropriate conditions, losses in one wavelength band can be efficiently compensated for in the presence of linear gain at the other wavelength band. We show the robustness of the compensation process and find families of chirped soliton solutions that exactly compensate loss and gain.

The physical setting considered is typically encountered in parametric processes that involve a wavelength band that falls beyond the ultraviolet or the infrared absorption edges of the material. Illustrative examples include BBO-based systems at wavelengths shorter than $0.2 \mu \mathrm{m}$ [11] and longer than $2 \mu \mathrm{m}$ [12], or OPO in PPLN out to $6 \mu \mathrm{m}$ under strong idler absorption [13]. Other important examples can arise with existing organics, semiconductors, and new molecularengineered materials with very large nonlinear coefficients [14]-[17]. On the other hand, broad-band gain at wavelengths around the third telecommunication window centered at 1.55 $\mu \mathrm{m}$ has been demonstrated in erbium-doped optically pumped $\mathrm{LiNbO}_{3}$ devices [18]. For example, gain rates of some $2 \mathrm{~dB} / \mathrm{cm}$ have been measured at the C-band of erbium amplification in $\mathrm{Er}: \mathrm{Ti}: \mathrm{LiNbO}_{3}$ channel waveguides diode-pumped at 1.48 $\mu \mathrm{m}$. Erbium-doped PPLN [19]-[20] and KTP [21] are also under current investigation. Another important related possibility is to operate self-frequency-doubling lasers (see [22], [23], and references therein) in the solitonic regime.

\section{EVOLUTION EQUATIONS}

In this paper, we focus on conditions suitable for spatial soliton formation in planar waveguides, but the results hold also for temporal solitons and the analysis can be extended to higher dimensional settings. The evolution of the slowly 
varying envelopes of $\mathrm{CW}$ light beams in a quadratic nonlinear medium under conditions for type I SHG in the presence of absorption losses at the second-harmonic frequency and linear gain at the fundamental frequency can be described by the reduced equations

$$
\begin{array}{r}
i \frac{\partial a_{1}}{\partial \xi}-\frac{\alpha_{1}}{2} \frac{\partial^{2} a_{1}}{\partial s^{2}}+a_{1}^{*} a_{2} \exp (-i \beta \xi)=i G a_{1} \\
i \frac{\partial a_{2}}{\partial \xi}-\frac{\alpha_{2}}{2} \frac{\partial^{2} a_{2}}{\partial s^{2}}-i \delta \frac{\partial a_{2}}{\partial s}+a_{1}^{2} \exp (i \beta \xi)=i \Gamma_{2} a_{2}
\end{array}
$$

where $a_{1}$ and $a_{2}$ are the normalized amplitudes of the fundamental frequency and second-harmonic waves, $\alpha_{1}=-1$, and $\alpha_{2}=-k_{1} / k_{2}$, with $k_{1,2}$ being the linear wavenumbers at both frequencies. For spatial solitons in all cases, $\alpha_{2} \simeq-0.5$, thus, in the numerical calculations, we set $\alpha_{2}=-0.5$. The parameter $\beta$ is the scaled wavevector mismatch $\beta=$ $k_{1} \eta^{2} \Delta k$, where $\Delta k=2 k_{1}-k_{2}$, with $\eta$ being a beamwidth. The parameter $\delta$ stands for the existence of Poynting vector walk-off. For our purposes here, it is convenient to consider configurations without walk-off; thus, here we set $\delta=0$. The scaled coordinates are $s=x / \eta$ and $\xi=z / 2 l_{d 1}$, with $l_{d 1}=k_{1} \eta^{2} / 2$ being the diffraction length at the fundamental frequency.

The function $G$ models a net linear gain at the fundamental frequency. In practice, the actual gain depends on the spatial and temporal dynamics of the population and depletion of the material energy levels and on the signal and pump features. With the aim to expose the essence of the process, here we consider a reduced $\mathrm{CW}$ saturationless model, with $G=$ $\Gamma_{1} \exp \left[-s^{2} / w_{g}^{2}\right]$, where $w_{g}$ is the transverse width of the amplifying region, and $\Gamma_{1}>0$. Unless otherwise stated, we set $w_{g} \gg 1$; thus, $G \approx \Gamma_{1}$, which corresponds to an amplifier much wider than the soliton. Full modeling can be performed to take into account the detailed dynamics of the light-matter interaction involved in the amplification [24]-[27], but the reduced model is expected to capture important aspects of the process. The parameter $\Gamma_{2}<0$ stands for spatially homogeneous linear absorption at the secondharmonic frequency.

In this paper, we concentrate on the normalized loss and gain coefficients in the range $\left|\Gamma_{1,2}\right|=0.1-2.0$. The actual loss and gain coefficient per unit length is given by $\gamma_{1,2}=\Gamma_{1,2} / 2 l_{d 1}$. Therefore, for a diffraction length of $l_{d 1} \sim 4 \mathrm{~mm}$ that is encountered in a material with refractive index $n \sim 2$ at $\lambda \sim 1.5 \mu \mathrm{m}$ with a beamwidth $\eta \sim 30 \mu \mathrm{m}$, setting $\left|\Gamma_{1,2}\right|=0.2$ yields $\left|\gamma_{1,2}\right| \sim 0.25 \mathrm{~cm}^{-1}$. In the case of linear propagation in a wide amplifying region (i.e., with $w_{g} \gg 1$ ) or in the presence of the spatially homogeneous absorption, such values give an actual linear gain or loss rate of some $\pm 2 \mathrm{~dB} / \mathrm{cm}$. In the presence of the quadratic nonlinearity, the actual rate depends on the dynamics of the wave evolution, as discussed below. For the above values, $\xi=5$ corresponds to a crystal length of about $4 \mathrm{~cm}$.

\section{Soliton Evolution with Gain AND Loss}

In the absence of gain and loss, the system (1)-(2) has families of stable stationary soliton solutions [28], [29], and

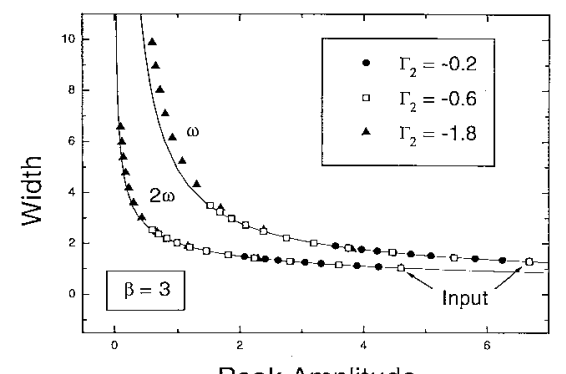

Peak Amplitude

Fig. 1. Evolution of the peak amplitude-width relation of the fundamental and second-harmonic waves that form the quadratic solitons under the influence of different second-harmonic linear losses. The discrete points show the values of the peak amplitudes and width of every propagation unit. The solid lines show the amplitude-width relation of the family of solitons existing in the absence of loss. Conditions: absorption coefficients $\Gamma_{2}=-0.2,-0.6$, and -1.8 ; wavevector mismatch $\beta=3$. The input signal is the corresponding soliton existing in the absence of loss with the energy flow $I=60$.

the total energy flow given by the Manley-Rowe relation

$$
I=I_{1}+I_{2}=\int\left(\left|a_{1}\right|^{2}+\left|a_{2}\right|^{2} d s\right.
$$

is conserved. Otherwise, letting $G=\Gamma_{1}$, the total energy flow varies at a rate

$$
\frac{d I}{d \xi}=2\left(\Gamma_{1} I_{1}+\Gamma_{2} I_{2}\right)
$$

It is worth recalling that, under the conditions considered here, $\Gamma_{1}>0$, whereas $\Gamma_{2}<0$. Thus, for given material parameters, the net gain/loss experienced by the solitons depends on their fundamental and second-harmonic energy content. Such content depends on the wavevector mismatch, on the total soliton energy, and on the dynamical soliton evolution.

By and large, under the influence of moderate gain and loss, solitons are expected to dynamically evolve, adapting themselves to the amplitude-width relation characteristic of their family. The question is whether, for the soliton families existing at each value of the wave vector mismatch, and for given loss/gain coefficients, such evolution takes place without large departures from the soliton shape. To investigate such evolution, we performed a comprehensive series of numerical experiments by solving the system (1)-(2) with a split-step Fourier algorithm for the soliton families existing at a variety of wavevector mismatches, with different loss/gain coefficients. Figs. 1-4 show representative examples of the outcome.

First, we start with the beam evolution in the presence of losses at the second-harmonic frequency, but negligible absorption at the fundamental (the case of small damping at both frequencies was discussed in [30]-[32]). Fig. 1 shows the typical evolution of the peak amplitudes and widths (FWHM) of the two-color beams forming the solitons propagated up to $\xi=10$. The discrete points correspond to the peak amplitudes and widths sampled at each propagation unit. The plot corresponds to the normalized wavevector mismatch $\beta=3$ which is representative of all experimental conditions near phase matching at positive phase mismatch, and the input signal is the exact soliton with energy flow $I=60$. This is a relatively high-energy soliton. The amplitude-width relation 


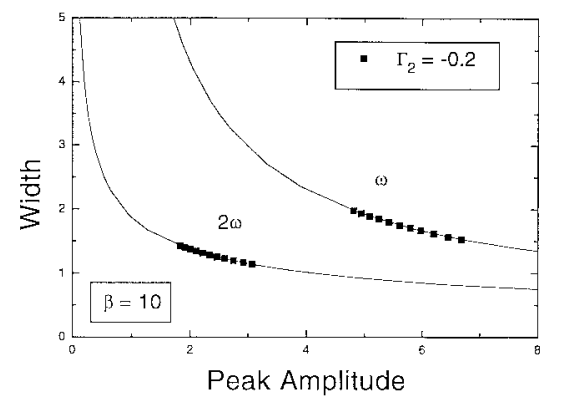

Fig. 2. Analogous to Fig. 1, but at the wavevector mismatch $\beta=10$. Conditions: absorption coefficient $\Gamma_{2}=-0.2$; input soliton energy flow $I=60$.

of the corresponding exact family of stationary solitons is also shown for the sake of direct comparison. At exact phase matching, all solitons existing in the absence of gain and loss are self-similar [28]-[30], and their peak amplitudes and widths are related by the expression $a_{\nu}(0) w_{\nu}^{2}=C_{\nu, D}$ where $C_{\nu, D}$, with $\nu=1,2$, is a constant and $D$ is the transverse dimensionality of the physical setting considered. Here we examine planar waveguides; hence, $D=1$. In such a case, one finds $C_{1,1} \simeq 9.54$, and $C_{2,1} \simeq 5.54$. Away from exact phase matching and when $\delta \neq 0$, the corresponding dependence can be found numerically [30].

Fig. 1 shows several interesting features. First, one observes that, with moderate loss rates at the second-harmonic frequency, the solitons decay adiabatically according to the amplitude-width relation characteristic of their family. This is because the beams are able to dynamically reshape and exchange energy with each other while the second-harmonic wave is linearly damped, so that they keep the proper amplitude-width relation and the proper fundamental/secondharmonic fraction of energy corresponding to their total soliton energy and soliton family. Notice that in Fig. 1 this is still the case for many propagation units, even for the high-absorption case $\Gamma_{2}=-1.8$, which for the above-mentioned typical experimental conditions corresponds to an actual linear loss rate of about $20 \mathrm{~dB} / \mathrm{cm}$.

One also observes in Fig. 1 that the separation between the sampled points (hence, the actual energy loss experienced by the waves) decreases with propagation distance. For the wavevector mismatch considered, when the total soliton energy decreases, so does the fraction of such energy carried by the second-harmonic wave [29]. Therefore, so does the effective energy loss given by (4). An analogous effect can be obtained for a fixed soliton energy by increasing the existing wavevector mismatch, as shown in Fig. 2. The plot corresponds to $\Gamma_{2}=-0.2$ at $\beta=10$. The decay rate is to be compared with the corresponding curve shown in Fig. 1. Notice that, in both cases, the input conditions are the exact solitons existing in the absence of losses at the corresponding value of $\beta$ with the energy flow $I=60$. Therefore, the input signals are different in each case.

Next, we discuss the robustness of the soliton evolution in the presence of losses at the second-harmonic frequency together with a compensating gain at the fundamental frequency band [33]. We note that a related scheme, except for

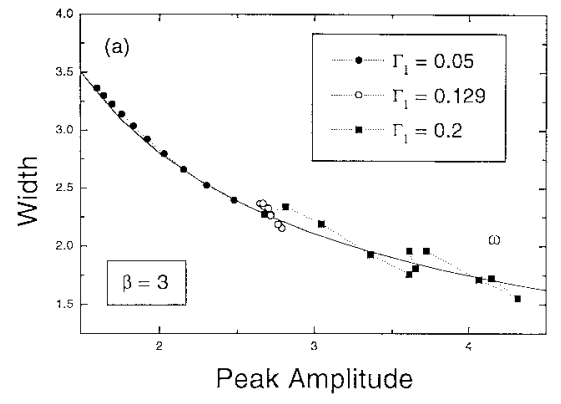

(a)

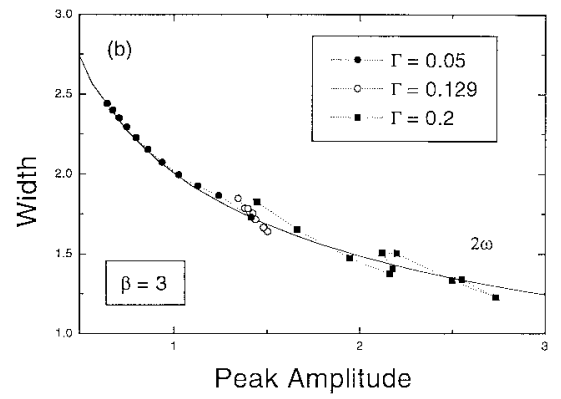

(b)

Fig. 3. Evolution of the peak amplitude-width relation of the solitons under the influence of second-harmonic losses and fundamental frequency gain. Features are the same as in Fig. 1. (a) Fundamental wave. (b) Second-harmonic. Conditions: absorption coefficient $\Gamma_{2}=-0.6$; gain coefficients $\Gamma_{1}=0.05$, 0.129 , and 0.2 ; wavevector mismatch $\beta=3$. The input signal is the corresponding soliton existing in the absence of loss with the energy flow $I=15$.

$\mathrm{CW}$ signals, namely losses at fundamental frequency and gain at the second-harmonic frequency, was considered recently in the context of nonlinear optical fiber amplifiers for cubic solitons [34]. Fig. 3 shows representative examples of the beam evolutions encountered under such conditions. The plot corresponds to the evolution of a soliton with energy flow $I=15$ propagating in a sample with a loss coefficient $\Gamma_{2}=-0.6$, and with different gain coefficients. At low values of the gain coefficient, the soliton experiences net losses, while with high gain rates amplification dominates. The plot shows that, in either case, the beams tend to evolve adiabatically, following to a large extent the amplitude-width relation of the families of solitons existing in the absence of gain and loss.

For a given wavevector mismatch, soliton energy, and second-harmonic absorption coefficient, there is an optimum gain rate at the fundamental frequency for which the overall losses and gain almost compensate for each other. For the conditions shown in Fig. 3, such gain coefficient amounts to some $\Gamma_{1} \simeq 0.13$. Under such conditions, the fundamental signal transfers rapidly the energy it gains by the amplification to its second-harmonic counterpart, in such a way that the overall energy is almost constant and the signal shape barely differs from an exact soliton of similar energy existing in the absence of gain and loss. Naturally, the energy exchange and continuous reshaping produces dispersive waves that are radiated away, but for moderate values of $\Gamma_{1,2}$ such radiation is very small. Higher energy solitons feature sharper profiles; therefore, under similar conditions, they tend to produce more dispersive waves. Yet, for moderate values of the loss and gain 


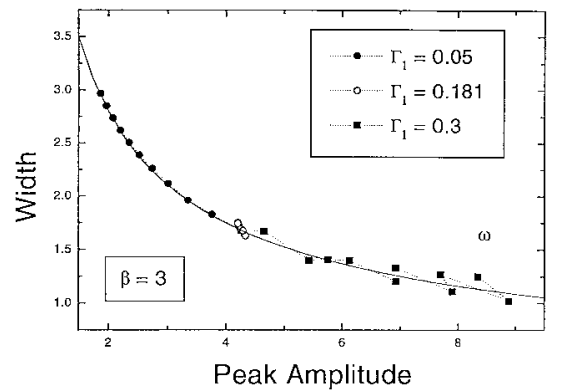

(a)

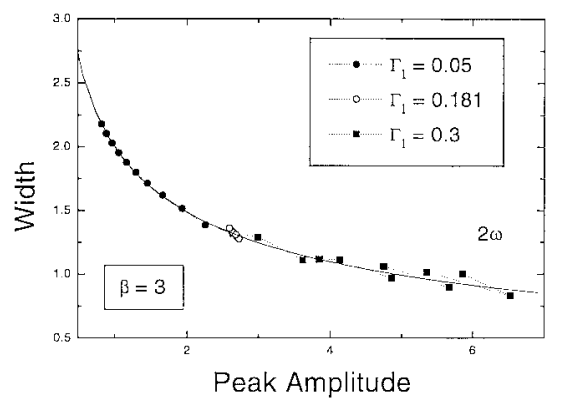

(b)

Fig. 4. Analogous to Fig. 3, but when the input signal is the soliton existing in the absence of losses with the energy flow $I=30$. Conditions: absorption coefficient $\Gamma_{2}=-0.6$; gain coefficients $\Gamma_{1}=0.05,0.181$, and 0.3 ; wavevector mismatch $\beta=3$.

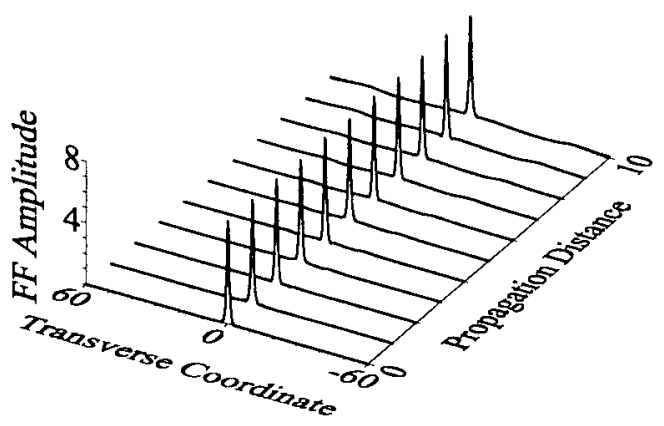

(a)

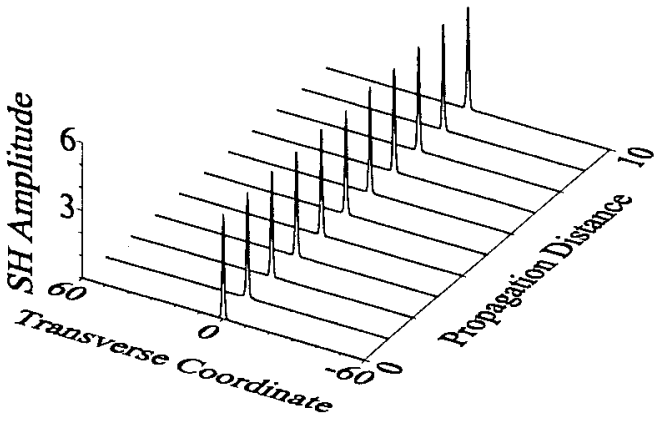

(b)

Fig. 5. Detailed light evolution in a typical case. Conditions: $\Gamma_{1}=0.226, \Gamma_{2}=-0.6, \beta=3, I=60$.

coefficients, an analogous behavior is obtained, as illustrated in Fig. 4. Fig. 5 shows the detailed signal evolution in a typical case. The generated radiation is so small, it is barely visible in the plot.

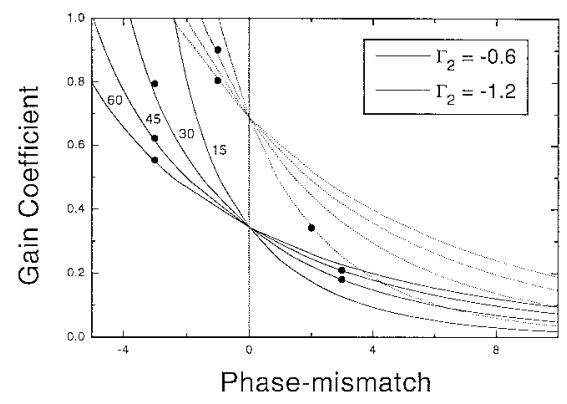

Fig. 6. Gain coefficient at the fundamental frequency required for loss compensation when the input signals are exact solitons, as a function of the wavevector mismatch, for different values of the total soliton energy and the second-harmonic absorption coefficient. Curves: estimates obtained using the properties of the solitons existing without gain and loss; discrete big dots: values calculated with the chirped solitary-wave solutions that exactly compensate gain and loss. The labels indicate the value of the soliton energy.

According to (4), exact loss-gain compensation at $\xi=0$ occurs for input signals that satisfy

$$
\Gamma_{1} I_{1}+\Gamma_{2} I_{2}=0
$$

Obviously, this does not mean that all input signals that satisfy the above condition propagate in a stationary way, because, in general, their dynamical evolution, governed by (1)-(2), rapidly modifies the $I_{1} / I_{2}$ relation required for (5) to hold. However, Figs. 3-5 suggest that, for given reasonable values of the wavevector mismatch $\beta$ and of the absorption coefficient $\Gamma_{2}$, (5) with $I_{1} / I_{2}$ calculated from the families of solitons existing in the absence of gain and loss can be used to estimate the gain rate for a given soliton energy (or, alternatively, the soliton energy for a given gain rate), required to obtain approximate stationary soliton signals. Notice that this is only so when the input signals are the solitons existing in the absence of gain and loss. Different estimates will result under other conditions, such as the typical up-conversion or down-conversion experimental setups with only fundamental or second-harmonic pump light, respectively.

Fig. 6 shows the values obtained of the gain coefficient as a function of the wavevector mismatch for different values of the soliton energy and the absorption coefficient. The labels indicate the value of the soliton energy $I=15,30$, 45 , and 60 . The solid and dashed curves are the values of $\Gamma_{1}$ calculated using the data corresponding to the families of solitons existing in the absence of gain and loss, and the few discrete dots show the values calculated with the chirped solitary-wave solutions discussed in Section IV that exactly compensate gain and loss. One observes in the selected cases shown that the difference between both values is very small. Because at positive $\beta$ most of the soliton energy is carried by the fundamental wave, a correspondingly small gain coefficient is required to compensate for second-harmonic absorption. The main conclusion to be drawn from Fig. 6 is that gain coefficients in the range experimentally demonstrated in diode-pumped Er: $\mathrm{LiNbO}_{3}$ samples [18] are required at positive wavevector mismatch for a variety of soliton energies and second-harmonic absorption coefficients. A much higher compensating gain would be required at negative $\beta$. 


\section{EXACT LOSS-GAIN COMPENSATION}

Guided by the results shown in the previous section, we seek stationary localized solitary-wave solutions of the system (1)(2) having the form $a_{\nu}(\xi, s)=U_{\nu}(s) \exp \left(i \kappa_{\nu} \xi\right)$. Because of the presence of gain and loss, the solutions should be chirped and, hence, $U_{\nu}(s)$ are complex functions. The parameters $\kappa_{\nu}$ are nonlinear wavenumber shifts induced by the wave interaction. Stationary solutions must have $\kappa_{2}=2 \kappa_{1}+\beta \pm \pi$. The complex coupled differential equations obeyed by the complex profiles $U_{\nu}(s)$ are

$$
\begin{aligned}
\frac{\alpha_{1}}{2} \frac{d^{2} U_{1}}{d s^{2}}+\kappa_{1} U_{1}-U_{1}^{*} U_{2} & =-i \Gamma_{1} U_{1} \\
\frac{\alpha_{2}}{2} \frac{d^{2} U_{2}}{d s^{2}}+\left(2 \kappa_{1}+\beta\right) U_{2}-U_{1}^{2} & =-i \Gamma_{2} U_{2} .
\end{aligned}
$$

We seek stationary solitary-wave solutions that keep constant not only the total energy flow but also the fundamental and second-harmonic energies separately. Let $U_{\nu}(s)=$ $S_{\nu}(s) \exp \left[i \phi_{\nu}(s)\right]$, where $S_{\nu}$ and $\phi_{\nu}$ are now real functions. Substitution into (1) and (2) and integration readily yields

$$
\frac{d I_{2}}{d \xi}=2 \int S_{1}^{2} S_{2} \sin \left(\phi_{2}-2 \phi_{1}\right) d s+2 \Gamma_{2} I_{2} .
$$

Therefore, because $\Gamma_{2} \neq 0$, one needs $\phi_{2} \neq 2 \phi_{1}$. Also, both the amplitude profiles $S_{\nu}$ and the phases $\phi_{\nu}$ ought to be symmetric functions of the transverse coordinates. Thus, the chirps $d \phi_{\nu} / d s$ are antisymmetric functions. The asymptotic values for the chirps far from the soliton peak may be obtained by linearizing (6) and (7). Assuming

$$
S_{\nu} \sim \exp \left(-\Omega_{\nu} s\right), \quad s \rightarrow \infty
$$

with $\Omega_{2}>2 \Omega_{1}$ and linearizing (6) and (7) gives

$$
\Omega_{\nu}^{2}=\frac{1}{\alpha_{\nu}}\left[-\kappa_{\nu} \pm \sqrt{\kappa_{\nu}^{2}+\Gamma_{\nu}^{2}}\right]
$$

and

$$
\left.\frac{d \phi_{\nu}}{d s}\right|_{s \rightarrow \infty}=\frac{\Gamma_{\nu}}{\alpha_{\nu} \Omega_{\nu}}
$$

However, the tails of the second-harmonic wave might be more complicated than implied by (9) when the nonlinear terms in (7) decay as $s \rightarrow \pm \infty$ with the same rate as the linear terms. Under these conditions, (11) only holds for the fundamental wave.

When $\beta$ is positive and large, one can obtain approximate analytical solitary-wave solutions of (1) and (2) as follows. Let $A_{2}=a_{2} \exp (-i \beta \xi)$. Under conditions of large positive phase-mismatch and negligible pump conversion, the secondharmonic field can be approximated by

$$
A_{2} \simeq \frac{1}{\beta+i \Gamma_{2}} a_{1}^{2} .
$$

Substitution into the equation for the fundamental wave yields

$$
i \frac{\partial q}{\partial \xi}-\frac{\alpha_{1}}{2} \frac{\partial^{2} q}{\partial s^{2}}+|q|^{2} q=i \Gamma_{1} q+i \epsilon|q|^{2} q
$$

where $q=\sqrt{\beta /\left(\beta^{2}+\Gamma_{2}^{2}\right)} a_{1}$, and $\epsilon=\Gamma_{2} / \beta$. Under the conditions where (12) is expected to hold, one has $\epsilon<0,|\epsilon| \ll 1$.
Equation (13) is a truncated cubic complex Ginzburg-Landau equation. A known pulse-like stationary solution of this equation is given by [35]

$$
q(\xi, s)=A \operatorname{sech}(B s) \exp \{i \kappa \xi+i d \operatorname{In}[A \operatorname{sech}(B s)]\}
$$

where

and

$$
\begin{aligned}
d & =\frac{3-\sqrt{9+8 \epsilon^{2}}}{2 \epsilon} \\
A & =\sqrt{\frac{3 \Gamma_{1}}{-2 \epsilon}} \\
B & =\sqrt{\frac{-\alpha_{1} \Gamma_{1}}{d}}
\end{aligned}
$$

$$
\kappa=\Gamma_{1} \frac{1-d^{2}}{2 d} .
$$

At arbitrary values of $\beta$, one can look for analytical solutions to (6) and (7) of the form

$$
S_{\nu}(s)=A_{\nu} R_{\nu}(s)
$$

and

$$
\phi_{\nu}(s)=d_{\nu} \operatorname{In}\left[S_{\nu}(s)\right]
$$

with $R_{\nu}=\operatorname{sech}^{2}(w s)$. Substitution into (6) and (7) readily yields

$$
\begin{aligned}
d_{2} & =2 d_{1}=\sqrt{3} \\
w & =\left[\frac{-\Gamma_{1}}{2 \sqrt{3} \alpha_{1}}\right]^{1 / 2} \\
\kappa_{1} & =\frac{\Gamma_{1}}{4 \sqrt{3}} \\
A_{1} & =\frac{\sqrt{7}}{2 a}\left|\Gamma_{1}\right|
\end{aligned}
$$

and

$$
A_{2}=\frac{\sqrt{7}}{2} \Gamma_{1}
$$

where $a=\exp \left[(1 / \sqrt{3}) \tan ^{-1}(5 / \sqrt{3})\right] \simeq 2.0429$. This zeroparameter solution exists for the special values of the material parameters that fulfill the conditions

$$
\begin{aligned}
& \frac{\Gamma_{1}}{\Gamma_{2}}=-a^{2} \\
& \frac{\alpha_{1}}{\alpha_{2}}=-2 a^{2}
\end{aligned}
$$

and

$$
\beta=-\frac{\Gamma_{1}}{2 \sqrt{3}}\left(1-\frac{2}{a^{2}}\right) .
$$

Equation (26) is a consequence of the fact that stationary solutions can only exist in the presence of both gain and loss. Equation (27) indicates that the above analytical solution only holds in the case of temporal solitons, when one of the waves experiences normal group-velocity dispersion (GVD) while the other wave experiences anomalous GVD.

To find solitary-wave solutions of (6) and (7) at arbitrary values of all the material parameters, including those that hold for the spatial solitons we primarily consider here, we solved numerically with a relaxation algorithm the system (6) and (7) 

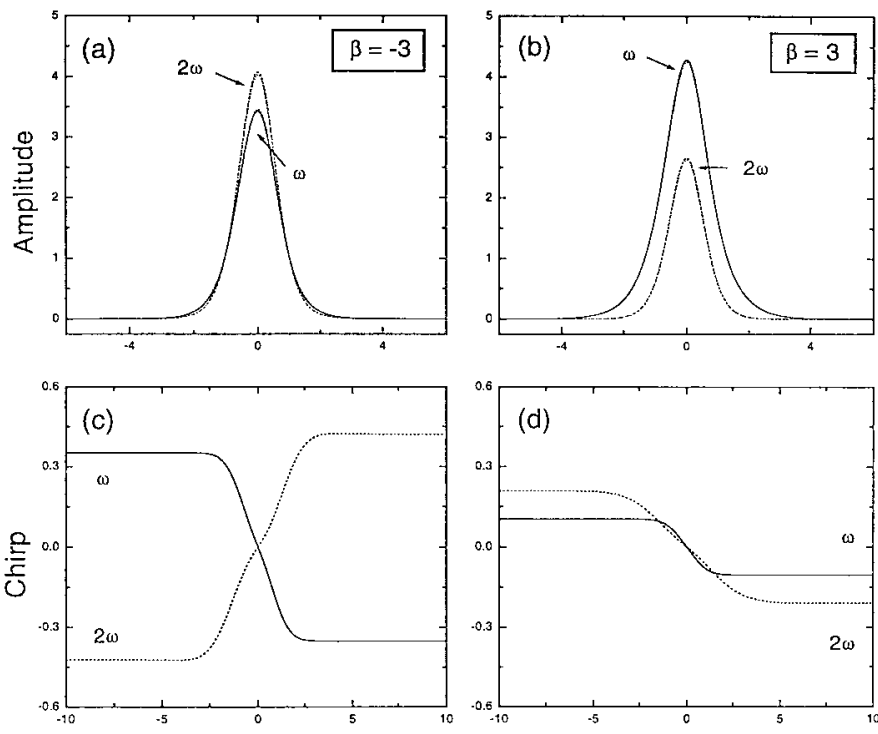

Transverse Coordinate

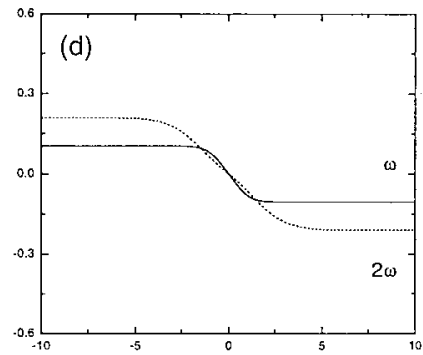

Transverse Coordinate

Fig. 7. Typical amplitude profile and chirp of solitary-wave solutions that exactly compensate gain and loss. (a), (c) $\beta=-3, \Gamma_{1}=0.75$. (b), (d) $\beta=3, \Gamma_{1}=0.181$. In both cases, $I \simeq 30$ and $\Gamma_{2}=-0.6$. Solid lines: fundamental frequency; dashed lines: second-harmonic. The dotted lines barely visible in (a) and (b) correspond to the amplitudes of the solitons existing with the same energy flow but in the absence of gain and loss.

together with (5). Fig. 7 shows two illustrative examples of the outcome of the calculations for representative values of the material parameters. The dotted lines barely visible in Fig. 7(a) and (b) correspond to the amplitudes of the solitons existing with the same energy flow in the absence of gain and loss. As mentioned above, one observes in the plot that the amplitudes of the solitary-wave solutions with and without gain and loss are almost identical. Fig. 7(c) and (d) shows the chirps of the solitary-wave solutions that exactly compensate gain and loss. For the solution shown in Fig. 7(a) and (c), which corresponds to the wavevector mismatch $\beta=-3$, (9) holds. Thus, (11) holds as well. However, this is not the case for the solitary wave shown in Fig. 7(b) and (d), which corresponds to a similar energy flow but for the positive wavevector mismatch $\beta=3$. In this case, (11) does not hold for the second-harmonic wave, as is clearly visible in Fig. 7(d).

The solitary-wave solutions of the model (6) and (7) cannot be stable under propagation because any perturbations to the fundamental beams far from the solution peak are amplified by the linear gain that they experience. Such background instability is suppressed when the amplifier transverse width $w_{g}$ is set to a finite value of the order of the soliton width, as can be done in practice by engineering the Er-doped transverse profiles or by proper shaping of the pump beam. Spatial spectral filtering schemes, similar to those appearing in the gain-bandwidth of erbium-doped fiber amplifiers [35], but implemented here in the spatial domain, constitute another interesting possibility for future research.

\section{CONCLUSIONS}

In summary, we discussed the robustness of soliton signals propagating in quadratic nonlinear media in the presence of moderate absorption and amplification in different frequency bands. We showed the signal reshaping under the action of linear absorption and gain, discussed the possibility of loss-gain compensation, and found new chirped solitary-wave solutions that theoretically allow for exact compensation. Results can be extended to include nonlinear absorption mechanisms, and spectrally filtered amplification schemes in spatial or temporal domains. Here we focused on conditions for typeI noncritically phase-matched second-harmonic generation, but we believe that the main results reported have direct implications for soliton propagation schemes in all parametric processes mediated by quadratic nonlinearities. The results presented show the feasibility of quadratic soliton propagation in novel materials, unexplored settings, and new wavelength bands which feature moderate absorption and suitable linear amplification at the different frequency bands involved.

\section{REFERENCES}

[1] G. I. Stegeman, D. J. Hagan, and L. Torner, " $\chi{ }^{(2)}$ cascading phenomena and their applications to signal processing, mode-locking, pulse compression and solitons," Opt. Quantum Electron., vol. 28, pp. 1691-1740, 1996.

[2] L. Torner, "Spatial solitons in quadratic nonlinear media," in Beam Shaping and Control with Nonlinear Optics, F. Kajzar and R. Reinisch Eds.. New York: Plenum, 1998, pp. 229-258.

[3] Yu. S. Kivshar, "Quadratic solitons: Past, present and future," in Advanced Photonics with Second-Order Optically Nonlinear Processes, A. D. Boardman, L. Pavlov, and S. Tanev, Eds. Dordrecht, The Netherlands: Kluwer, 1998, pp. 451-475.

[4] R. Schiek, Y. Baek, and G. I. Stegeman, "One-dimensional spatial solitary waves due to cascaded second-order nonlinearities in planar waveguides," Phys. Rev. E, vol. 53, pp. 1138-1141, 1996.

[5] W. E. Torruellas, Z. Wang, D. J. Hagan, E. W. VanStryland, G. I. Stegeman, L. Torner, and C. R. Menyuk, "Observation of twodimensional quadratic solitons in a quadratic medium," Phys. Rev. Lett., vol. 74, pp. 5036-5039, 1995.

[6] M. T. G. Canva, R. A. Fuerst, D. M. Baboiu, G. I. Stegeman, and G. Assanto, "Quadratic spatial soliton generation by seeded downconversion of a strong harmonic pump beam," Opt. Lett., vol. 22, pp. 1683-1685, 1997.

[7] R. A. Fuerst, R. R. Malendevich, C. G. Treviño-Palacios, and G. I. Stegeman, CREOL, University of Central Florida, Orlando, FL, USA; Ch. Bosshard and P. Günter, Swiss Federal Institute of Technology, Zurich, Switzerland, private communication, Dec. 1998.

[8] A. Barthélémy, B. Bourliaguet, and V. Couderc, G. W. Ross, P. G. R Smith, D. C. Hanna, and C. De Angelis, private communication, Jan. 1999.

[9] P. Di Trapani, G. Valiulis, W. Chinaglia, and A. Andreoti, "Twodimensional spatial solitary waves from traveling-wave parametric amplification of the quantum noise," Phys. Rev. Lett., vol. 80, pp. 265-268, 1998.

[10] P. Di Trapani, D. Caironi, G. Valiulis, A. Dubietis, R. Danielius, and A. Piskarskas, "Observation of temporal solitons in second-harmonic generation with tilted pulses," Phys. Rev. Lett., vol. 81, pp. 570-573, 1998.

[11] G. C. Bhar, U. Chatterjee, A. M. Rudra, P. Kumbhakar, R. K. Route, and R. S. Feigelson, "Generation of tunable 187.9-196-nm radiation in $\beta-\mathrm{BaB}_{2} \mathrm{O}_{4}$, , Opt. Lett., vol. 22, 1606-1608, 1997.

[12] Y. X. Fan, R. C. Eckardt, R. L. Byer, J. Nolting, and R. Wallenstein, "Visible $\mathrm{BaB}_{2} \mathrm{O}_{4}$ optical parametric oscillator pumped at $355 \mathrm{~nm}$ by a single-axial-mode pulsed source," Appl. Phys. Lett., vol. 53, pp. 2014-2016, 1988.

[13] L. Lefort, K. Puech, G. W. Ross, Y. P. Svirko, and D. C. Hanna, "Optical parametric oscillation out to $6.3 \mu \mathrm{m}$ in periodically poled lithium niobate under strong idler absorption," Appl. Phys. Lett., vol. 73, pp. 1610-1612, 1998.

[14] I. Ledoux and J. Zyss, "Molecular nonlinear optics: Fundamentals and applications," in Novel Optical Materials and Applications, I. C. Khoo, F. Simoni, and C. Umeton, Eds. New York: Wiley, 1997, pp. 1-48.

[15] Ch. Bosshard, G. Knöpfle, P. Prêtre, S. Follonier, C. Serbutoviez, and P. Günter, "Molecular crystals and polymers for nonlinear optics," Opt. Eng., vol. 34, pp. 1951-1960, 1995. 
[16] U. Meier, M. Bösch, Ch. Bosshard, F. Pan, and P. Günter, "Parametric interactions in the organic 4-N, N-dimethylamino- $44^{\prime}-\mathrm{N}^{\prime}$-methylstilbazolium tosylate at telecommunication wavelengths," J. Appl. Phys., vol. 83, pp. 3486-3489, 1998.

[17] P. Schunemann, "Nonlinear crystals provide high power for the mid IR," Laser Focus World, vol. 35, no. 4, pp. 85-90, Apr. 1999.

[18] I. Baumann, S. Bosso, R. Brinkmann, R. Corsini, M. Dinand, A. Greiner, K. Schäfer, J. Süchtig, W. Sohler, H. Suche, and R. Wessel, "Er-doped integrated optical devices in $\mathrm{LiNbO}_{3}$," IEEE J. Select. Topics Quantum Electron., vol. 2, pp. 355-365, 1996.

[19] V. Bermúdez, J. Capmany, J. García-Solé, and E. Diéguez, "Growth and second-harmonic generation characterization of $\mathrm{Er}^{3+}$ doped bulk periodically poled $\mathrm{LiNbO}_{3}$," Appl. Phys. Lett., vol. 73, pp. 593-595, 1998.

[20] W. Sohler, Angewandte Physik, Universität Paderborn, Germany, private communication, Apr. 1999.

[21] K.-M. Wang, B.-R. Shi, N. Cue, Y.-Y. Zhu, R.-F. Xiao, F. Lu, W. Li, and Y.-G. Liu, "Waveguide laser film in erbium-doped $\mathrm{KTiOPO}_{4}$ by pulsed laser deposition," Appl. Phys. Lett., vol. 73, pp. 1020-1022, 1998.

[22] J. Bartschke, R. Knappe, K.-J. Boller, and R. Wallenstein, "Investigation of efficient self-frequency-doubling Nd: YAB lasers," IEEE J. Quantum Electron., vol. 33, pp. 2295-2300, 1997.

[23] D. Jaque, J. Capmany, J. García-Solé, Z. D. Luo, and A. D. Jiang, "Continuous-wave laser properties of the self-frequency-doubling $\mathrm{YAl}_{3}\left(\mathrm{BO}_{3}\right)_{4}$ : Nd crystal," J. Opt. Soc. Amer. B, vol. 15, pp. 1656-1662, 1998.

[24] M. Dinand and W. Sohler, "Theoretical modeling of optical amplification in Er-doped Ti: $\mathrm{LiNbO}_{3}$ waveguides," IEEE J. Quantum Electron., vol. 30, pp. 1267- 1276, 1994.

[25] R. Brinkmann, I. Baumann, M. Dinand, W. Sohler, and H. Suche, "Erbium-doped single- and double-pass $\mathrm{Ti}: \mathrm{LiNbO}_{3}$ waveguide amplifiers," IEEE J. Quantum Electron., vol. 30, pp. 2356-2360, 1994.

[26] D. Scarano and I. Montrosset, "Transversal mode competition in erbiumdoped $\mathrm{Ti}$ : $\mathrm{LiNbO}_{3}$ waveguide lasers," IEEE J. Quantum Electron., vol 32, pp. 628-637, 1996.

[27] D. L. Veasey, J. M. Gary, J. Amin, and J. A. Aust, "Time-dependent modeling of erbium-doped waveguide lasers in lithium niobate pumped at 980 and $1480 \mathrm{~nm}, "$ IEEE J. Quantum Electron., vol. 33, pp. 1647-1662, 1997.
[28] A. V. Buryak and Yu. S. Kivshar, "Solitons due to second-harmonic generation," Phys. Lett. A, vol. 197, pp. 407-412, 1995.

[29] L. Torner, "Stationary solitary waves with second-order nonlinearities," Opt. Commun., vol. 114, pp. 136-140, 1995.

[30] L. Torner, D. Mihalache, D. Mazilu, and N. N. Akhmediev, "Stability of spatial solitary waves in quadratic media," Opt. Lett., vol. 20, pp. 2183-2185, 1995

[31] K. Hayata and M. Koshiba, "Theory of stationary solitary waves generated by optical parametric interactions in the presence of Kerrtype nonlinearities and dissipations," J. Opt. Soc. Amer. B, vol. 12, pp. 2288-2295, 1995.

[32] B. A. Malomed, D. Anderson, A. Berntson, M. Florjancyk, and M. Lisak, "Decay of parametric solitons in a lossy medium with quadratic nonlinearity," Pure Appl. Opt., vol. 5, pp. 941-946, 1996.

[33] L. Torner, "Amplification of quadratic solitons," Opt. Commun., vol. 154, pp. 59-64, 1998

[34] G. D. Peng, B. A. Malomed, and P. L. Chu, "Soliton amplification and reshaping by a second-harmonic-generating nonlinear amplifier," J. Opt. Soc. Amer. B, vol. 15, pp. 2462-2472, 1998.

[35] N. N. Akhmediev and A. Ankiewicz, Solitons. London, U.K.: Chapman and Hall, 1997, ch. 13.

Lluis Torner (M'92), photograph and biography not available at the time of publication.

Jochen Dörring, photograph and biography not available at the time of publication.

Juan P. Torres, photograph and biography not available at the time of publication. 